\title{
La motivación laboral y el compromiso organizacional
}

\section{Job motivation and organizational commitment}

\author{
Miguel Puma ${ }^{1}$; Edwin Estrada ${ }^{2}$
}

\section{RESUMEN}

El presente trabajo de investigación tuvo como objetivo determinar la relación que existe entre la motivación laboral y el compromiso organizacional de los trabajadores de la Corte Superior de Justicia de Madre de Dios. El enfoque de investigación fue cuantitativo, el diseño fue no experimental y el nivel correlacional. La muestra estuvo constituida por 80 trabajadores de la Corte Superior de Justicia de Madre de Dios, cantidad que fue obtenida mediante un muestreo probabilístico. Los instrumentos utilizados para la recolección de datos fueron el Cuestionario de Motivación elaborado por Sulca (2015) y el Cuestionario de Compromiso Organizacional adaptado por Rivera (2010). Los resultados hallados indican que existe una correlación positiva alta entre la motivación laboral y el compromiso organizacional. El coeficiente de correlación rho de Spearman de es 0,759 con un $\mathrm{p}$-valor inferior al nivel de significancia $(\mathrm{p}=0,000<0,05)$. Finalmente se concluye que mientras los trabajadores presenten altos niveles de motivación labora, su compromiso hacia la labor que realizan será mayor.

Palabras clave: Motivación laboral, compromiso organizacional, desempeño.

\section{ABSTRACT}

This research work aimed to determine the relation between work motivation and the organizational commitment of the workers of the Superior Court of Justice of Madre de Dios. The research approach was quantitative, the design was non-experimental and the correlational level. The sample consisted of 80 workers of the Superior Court of Justice of Madre de Dios, an amount that was obtained through a probabilistic sampling. The instruments used for data collection were the Job Motivation Questionnaire prepared by Sulca (2015) and the Organizational Commitment Questionnaire adapted by Rivera (2010). The results found indicate that there is a high positive correlation between work motivation and organizational commitment. Spearman's rho correlation coefficient of is 0.759 with a p-value below the level of significance $(p=0,000<0.05)$. Finally, it is concluded that as long as the workers present high levels of work motivation, their commitment to the work they do will be greater.

Keywords: Job motivation, organizational commitment, performance.

1. Magíster en Gestión Pública. Docente de la Universidad Nacional Amazónica de Madre de Dios. E-mail: pumaabg@gmail.com

2. Doctor en Educación. Docente de la Universidad Nacional Amazónica de Madre de Dios. E-mail: edwin5721@ outlook.com 


\section{INTRODUCCIÓN}

En la actualidad toda organización busca que sus trabajadores se encuentren motivados y comprometidos con la labor que realizan puesto que son ellos los que realizan cotidianamente una serie de acciones, de modo que esta permita alcanzar sus objetivos y en definitiva su misión. Como se sabe, "cuando una persona se encuentra motivada, se orienta hacia la meta, persiste y sobrepasa las dificultades que limitan su camino hacia el logro de dicha meta" (Pérez, 2014).

Sin embargo, en la Corte Superior de Justicia de Madre de Dios se ha podido percibir que los trabajadores presentan niveles de motivación que no son del todo favorables puesto que la jornada laboral es amplia y la remuneración no compensa el trabajo realizado. Del mismo modo casi no existen políticas que promuevan el reconocimiento del cumplimiento de las funciones de los trabajadores, lo que genera en ellos descontento y poca identificación, lo cual afectaría el compromiso hacia su institución, lo que se ve en la constante rotación del personal. Por otra parte, no existe mucha preocupación porque la alta dirección realice talleres o charlas para que los trabajadores se sientan más motivados y comprometidos con la labor que realizan, lo cual repercutiría en el logro eficiente y eficaz de los objetivos que la institución se ha propuesto.

Como se sabe, ambas variables son importantes dentro de una institución puesto que direccionan el comportamiento, actitud y dedicación que puedan manifestar los trabajadores para mejorar su desempeño y la productividad lo cual es la razón de ser de toda organización. Según Salazar, et al., (2009) "el comportamiento de un trabajador no es una resultante de los factores organizacionales existentes, sino que depende de la percepción que posee el trabajador de ellos" (p.68).

Como antecedentes de investigación tenemos a Córdova (2018) quien halló que existe una estrecha relación positiva estadísticamente significativa de alta intensidad entre la motivación labo- ral y el compromiso organizacional ( $r h o=0,898$; $\mathrm{p}=0,000<0,05)$. Ello significa que la motivación laboral promueve en los trabajadores al deseo de ser parte de la institución, permanecer como integrante, sentir altos niveles de identificación con la misma y mediante su accionar y ser prescriptor de ésta con su círculo social.

Asimismo se encuentran García y Gonzáles (2018) quienes hallaron que la motivación laboral se relaciona con el compromiso organizacional, en los trabajadores de la Municipalidad Provincial de Sánchez Carrión, 2018. El coeficiente de correlación rho de Spearman fue de $0.605 \mathrm{y}$ el p-valor igual a $0.000(\mathrm{p}<0.05)$. Los datos expuestos indican que cuanto más motivados se encuentran los trabajadores, mayor será el compromiso que tengan hacia la municipalidad.

Por otra parte está Vega (2017) quien determinó que existe una relación directa entre motivación laboral y compromiso organizacional en el personal del área de Seguridad de la Información y prevención del Fraude de la empresa Telefónica Ingeniería de seguridad, 2015.

Ello debido a que el valor del coeficiente de correlación de Spearman fue 0,296, con un p-valor de 0,009 que resulta menor al valor 0,05 de la significancia. Esto significa que en la medida que los trabajadores reciban niveles adecuados de motivación, se pondrán la camiseta y darán todo de ellos para alcanzar los objetivos institucionales trazados.

Una vez de haber revisado los antecedentes procederemos a definir ambas variables de estudio. La motivación para Vargas (2012) "es una característica de la psicología humana que contribuye al grado de compromiso de la persona; es un proceso que ocasiona, activa, orienta, dinamiza y mantiene el comportamiento de los individuos hacia la realización de objetivos esperados" (p. 14). Otra definición importante la realizan Herrera y Zamora (2014) quienes señalan que la motivación es "La motivación es un proceso autoenergético de la persona que ejerce una atrac- 
ción hacia un objetivo que supone una acción por parte del sujeto y permite aceptar el esfuerzo requerido para conseguirlo" (p.126).

En el campo del comportamiento organizacional, se concibe a la motivación como "un conjunto de fuerzas que actúan sobre una persona o en su interior y provocan que se comporte de una forma específica, encaminadas hacia una meta" (Hellriegel y Slocum, 2004, p.117). En el contexto laboral, la motivación comprende tanto los procesos individuales que llevan a un trabajador a actuar y que se vinculan con su desempeño y satisfacción en la empresa, como los procesos organizacionales que influyen para que tanto los motivos del trabajador como los de la empresa vayan en la misma dirección. (Olivares y Gonzáles, 2014).

De acuerdo a Herzberg, Mausner y Snyderman (1959) la motivación laboral presenta dos factores: motivacional e higiene. El primero guarda relación con el entorno físico que le rodea a la persona y tiene que ver con las condiciones en las cuales realizan su trabajo las cuales pueden ser relaciones con sus superiores y compañeros, prestigio, condiciones laborales, políticas de la organización así como condiciones de seguridad (Foncubierta y Sánchez, 2019). En cuanto al segundo factor (higiene), se relaciona de forma directa con los niveles de satisfacción que pueda sentir, los estímulos y reconocimientos que reciben, la oportunidad de seguir perfeccionando profesionalmente así como la autorrealización personal (Marín y Placencia, 2017).

Con relación al compromiso organizacional, Robbins (2004), indica que es el grado en que un trabajador se encuentra identificado con una determinada organización así como con sus metas, deseando mantener su permanencia en ella, es decir, al mantener un grado adecuado de compromiso organizacional, el trabajador tendrá mayor sentido de identificación y pertenencia con la organización donde labora.
Del mismo modo, Meyer y Allen (1991) lo definen como un estado psicológico que caracteriza la relación del trabajador con la organización donde labora y que influye en su decisión de mantener o no su condición de miembro de esa organización. Los mencionados autores afirman que el compromiso organizacional presenta 3 componentes: afectivo, de continuidad y normativo. El compromiso afectivo puede definirse como el lazo emocional que el trabajador crea con la institución donde labora. Asimismo, se relaciona con la sensación de las características subjetivas y objetivas de la institución, siendo necesaria para alcanzar niveles adecuados de satisfacción, motivación y éxito (Neves, et al., 2018). Con relación al compromiso de continuidad se vincula con la percepción de los trabajadores sobre lo que los costaría si dejarían de trabajar en la institución (Meyer y Allen, 1991). Por último, el compromiso normativo se refiere a la obligación moral que tiene el trabajador de permanecer en la institución donde labora debido a que considera que ella invirtió en él, dándole ciertas prestaciones que generan sentimientos de correspondencia (Chiang, Gómez y Wackerling, 2016). De acuerdo a Estrada (2020) "el compromiso organizacional se relaciona directamente con el sentido de pertenencia y la responsabilidad que posee cada persona con el lugar donde labora. Es trascendental tener trabajadores comprometidos y eficaces, en tanto que proporcionarían una alta eficacia y eficiencia organizacional" (p.133).

El aporte del presente estudio radica en que podremos conocer cuán motivados se encuentran los trabajadores frente a las labores que desempeñan diariamente y en qué medida se encuentran comprometidos con los objetivos estratégicos que presenta la institución. Del mismo modo, a partir de los resultados que se obtengan la Alta Dirección podrá plantear acciones de mejora lo que repercutirá en la imagen, objetivos y por sobre todo en los resultados de la institución que se proponga hacerla. Asimismo, a partir de éste trabajo se propone analizar la relación existente entre motivación laboral y el compromiso para poder establecer cómo es esa relación, determi- 
nando si con niveles altos de motivación en el trabajo se puede lograr trabajadores comprometidos, incorporando dichos conceptos por parte de la institución en sus políticas de recursos humanos.

Por lo expuesto, planteamos como problema de investigación: ¿Qué relación existe entre la motivación laboral y el compromiso organizacional de los trabajadores de la Corte Superior de Justicia de Madre de Dios? Para responder al problema planteamos como hipótesis general: Existe relación directa y significativa entre la motivación laboral y el compromiso organizacional de los trabajadores de la Corte Superior de Justicia de Madre de Dios. Finalmente, el objetivo general fue determinar la relación que existe entre la motivación laboral y el compromiso organizacional de los trabajadores de la Corte Superior de Justicia de Madre de Dios.

\section{MÉTODOS Y MATERIALES}

El enfoque de investigación fue cuantitativo y el diseño fue no experimental ya que las variables de estudio no fueron manipuladas deliberadamente, sino se observaron los fenómenos tal como se dieron en su entorno y luego fueron analizados (Hernández, Fernández y Baptista, 2014). Por otra parte, el nivel de investigación fue correlacional puesto que nos interesaba descubrir las relaciones que existen entre las variables analizadas.

La población de estudio estuvo conformada por 100 trabajadores de la Corte Superior de Justicia de Madre de Dios, entre varones y mujeres y la muestra fue conformada por 80 trabajadores. Dicha cantidad fue hallada mediante un muestreo probabilístico aleatorio con un nivel de confianza del $95 \%$ y un margen de error del 5\%.

Con relación a la técnica de recolección de datos, se hizo uso de la encuesta y los instrumentos fueron el Cuestionario de Motivación de Sulca (2015) y el Cuestionario de Compromiso Orga- nizacional de Rivera (2010), los cuales fueron adaptados. Con la finalidad de conocer las propiedades psicométricas de ambos instrumentos se realizó la validación de contenido, mediante el juicio de expertos, y la confiabilidad, mediante una prueba piloto.

El Cuestionario de Motivación consta de 16 ítems, fue estructurado mediante una escala de Likert (totalmente en desacuerdo, en desacuerdo, indeciso, de acuerdo y totalmente de acuerdo) y se divide en dos dimensiones: factor motivador y factor higiene. La escala de calificación de la variable motivación laboral presenta $3 \mathrm{ca}$ tegorías: bajo (16-37), medio (28-58) y alto (5980). En cuanto a la dimensión factor motivador, sus categorías también son bajo (7-16), medio (17-26) y alto (27-35). De la misma manera, la dimensión factor higiene, presenta 3 categorías: bajo (9-21), medio (22-33) y alto (34-45). Por otra parte, el cuestionario presenta un índice de validez de 0,80 lo cual significa que es adecuado y el coeficiente de confiabilidad Alfa de Cronbach fue de $\alpha=0,85$ lo cual nos permitió inferir que es altamente confiable.

Con relación al Cuestionario de Compromiso Organizacional, consta de 21 ítems, presenta una escala de Likert (Totalmente en desacuerdo, en desacuerdo, indeciso, de acuerdo y totalmente de acuerdo) y se divide en 3 dimensiones: compromiso afectivo, compromiso de continuidad y compromiso normativo. La escala de calificación de la variable compromiso organizacional presenta 3 categorías: bajo (21-42), medio (43-65) $y$ alto (66-84). En cuanto a la dimensión compromiso afectivo, sus categorías son bajo (8-16), medio (17-25) y alto (26-32). Con relación a la dimensión compromiso de continuidad, sus categorías también son bajo (7-14), medio (15-22) y alto (23-28). Respecto a la dimensión compromiso normativo, también tiene 3 categorías: bajo (6-12), medio (13-19) y alto (20-24). El índice de validez del cuestionario fue de 0,80 y un coeficiente de confiabilidad Alfa de Cronbach de $\alpha=0,84$ lo que indica que presenta una adecuada validez y una alta confiabilidad respectivamente. 
El procesamiento de los datos se realizó a través del programa Microsoft Excel 2013 ya que ahí se elaboró la base de datos y luego se trasladó al $\mathrm{Pa}$ quete Estadístico para las Ciencias Sociales SPSS versión 22. Para el análisis descriptivo se usaron las frecuencias y los porcentajes y para la prueba de hipótesis se recurrió a la prueba no paramétrica rho de Spearman puesto que las variables son ordinales.
Por último, con la finalidad de garantizar la seguridad y confidencialidad de los datos recogidos, se entregó a cada uno de los participantes una carta de consentimiento informado en la cual se le informó sobre el propósito de la investigación para así obtener su consentimiento voluntario y así pueda proporcionar información a la presente investigación.

\section{RESULTADOS}

\section{Tabla $N^{\circ} 1$. Niveles de motivación laboral}

\begin{tabular}{lcccc}
\hline Categoría & $\mathrm{n}$ & $\%$ & $\%$ válido & $\%$ acumulado \\
\hline Baja & 6 & 7,5 & 7,5 & 7,5 \\
Media & 44 & 55,0 & 55,0 & 62,5 \\
Alta & 30 & 37,5 & 37,5 & 100,0 \\
Total & 80 & 100,0 & 100,0 &
\end{tabular}

De acuerdo a la tabla 1 la mayoría de trabajadores de la Corte Superior de Justicia de Madre de Dios (55\%) presentan un nivel de motivación laboral medio, el 37,5\% presenta un nivel de motivación laboral alto y el 7,5\% presenta un nivel bajo.

Tabla $\mathrm{N}^{\circ}$ 2. Niveles de compromiso organizacional

\begin{tabular}{lcccc}
\hline Categoría & $\mathrm{n}$ & $\%$ & $\%$ válido & $\%$ acumulado \\
\hline Bajo & 3 & 3,8 & 3,8 & 3,8 \\
Medio & 18 & 22,5 & 22,5 & 26,3 \\
Alto & 59 & 73,8 & 73,8 & 100,0 \\
Total & 80 & 100,0 & 100,0 &
\end{tabular}

De acuerdo a la tabla 2, el 73,8\% de trabajadores de la Corte Superior de Justicia de Madre de Dios presentan un nivel de compromiso organizacional alto, el 22,5\% presenta un nivel medio y sólo el 3,8\% presenta un nivel de compromiso organizacional bajo.

Tabla N ${ }^{\circ}$ 3. Correlación entre la motivación laboral y el compromiso organizacional

\begin{tabular}{cclcc}
\hline & & & $\begin{array}{c}\text { Motivación } \\
\text { laboral }\end{array}$ & $\begin{array}{c}\text { Compromiso } \\
\text { organizacional }\end{array}$ \\
\hline \multirow{2}{*}{$\begin{array}{c}\text { Rho de } \\
\text { Spearman }\end{array}$} & Motivación & Coeficiente de correlación & 1,000 &, $759^{-}$ \\
\cline { 3 - 4 } & laboral & Sig. (bilateral) & &, 000 \\
\cline { 3 - 4 } & $\mathrm{N}$ & 80 & 80
\end{tabular}

**. La correlación es significativa en el nivel 0,01 ( 2 colas). 
En la tabla 3 se puede observar que el coeficiente de correlación rho de Spearman entre las variables motivación laboral y compromiso organizacional es de 0,759 con un p-valor inferior al nivel de significancia $(\mathrm{p}=0,000<0,05)$. Por lo expuesto, se concluye que existe una correlación alta, directa y significativa entre las variables analizadas. Esto quiere decir que mientras los trabajadores presenten altos niveles de motivación, mayor será su nivel de compromiso hacia la organización donde trabajan y viceversa.

Tabla N ${ }^{\circ}$. Correlación entre la motivación laboral y la dimensión compromiso afectivo

\begin{tabular}{cclcc}
\hline & & & $\begin{array}{c}\text { Motivación } \\
\text { laboral }\end{array}$ & $\begin{array}{c}\text { Compromiso } \\
\text { afectivo }\end{array}$ \\
\hline \multirow{2}{*}{\begin{tabular}{c} 
Rho de \\
\multirow{2}{*}{ Spearman }
\end{tabular}} & Motivación & Coeficiente de correlación & 1,000 &, $652^{* *}$ \\
\cline { 3 - 5 } & laboral & Sig. (bilateral) & - &, 000 \\
& & $\mathrm{~N}$ & 80 & 80 \\
\hline
\end{tabular}

**. La correlación es significativa en el nivel 0,01 ( 2 colas).

De acuerdo a la tabla 4, existe una correlación moderada, directa y significativa entre la motivación laboral y la dimensión compromiso afectivo. El coeficiente de correlación rho de Spearman es de 0,652 con un $\mathrm{p}$-valor inferior al nivel de significancia $(\mathrm{p}=0,000<0,05)$. Esto significa que si un trabajador presenta altos niveles de motivación, existirá un vínculo emocional a la organización donde labora y disfrutará su permanencia.

Tabla $N^{\circ}$. Correlación entre la motivación laboral y la dimensión compromiso de continuidad

\begin{tabular}{cclcc}
\hline & & & $\begin{array}{c}\text { Motivación } \\
\text { laboral }\end{array}$ & $\begin{array}{c}\text { Compromiso de } \\
\text { continuidad }\end{array}$ \\
\hline \multirow{2}{*}{$\begin{array}{c}\text { Rho de } \\
\text { Spearman }\end{array}$} & Motivación & Coeficiente de correlación & 1,000 &, $579^{-}$ \\
& laboral & Sig. (bilateral) & &, 000 \\
& & $N$ & 80 & 80 \\
& $* *$ La correlación es significativa en el nivel 0,01 (2 colas). &
\end{tabular}

Según la tabla 5, existe una correlación moderada, directa y significativa entre la motivación laboral y la dimensión compromiso de continuidad. El coeficiente de correlación rho de Spearman es de 0,579 con un $\mathrm{p}$-valor inferior al nivel de significancia $(\mathrm{p}=0,000<0,05)$. Esto quiere decir que mientras existan altos niveles de motivación en los trabajadores, mayor será el deseo de permanecer trabajando en la organización, puesto que sentirán que son parte de ella.

Tabla $N^{\circ}$ 6. Correlación entre la motivación laboral y la dimensión compromiso normativo

\begin{tabular}{cclcc}
\hline & & & $\begin{array}{c}\text { Motivación } \\
\text { laboral }\end{array}$ & $\begin{array}{c}\text { Compromiso } \\
\text { nomativo }\end{array}$ \\
\hline \multirow{2}{*}{$\begin{array}{c}\text { Rho de } \\
\text { Spearman }\end{array}$} & Motivación & Coeficiente de correlación & 1,000 &, $751^{* *}$ \\
& laboral & Sig. (bilateral) & &, 000 \\
& $N$ & 80 & 80
\end{tabular}

**. La correlación es significativa en el nivel 0,01 ( 2 colas).

En la tabla 6 podemos ver que existe una correlación alta, directa y significativa entre la motivación laboral y el compromiso normativo. El coeficiente de correlación rho de Spearman es de 0,751 y el 
$\mathrm{p}$-valor menor que el nivel de significancia planteado previamente $(\mathrm{p}=0,000<0,05)$. Esto significa que si los trabajadores logran estar altamente motivados, tendrán actitudes de lealtad y gratitud hacia la organización y se sentirán identificado con los fines que ella busque alcanzar.

\section{DISCUSIÓN}

El presente trabajo de investigación tuvo como objetivo determinar la relación que existe entre la motivación laboral y el compromiso organizacional de los trabajadores de la Corte Superior de Justicia de Madre de Dios, 2017.

Un primer hallazgo es que el 55\% de trabajadores de la Corte Superior de Justicia de Madre de Dios, 2017 presentan un nivel de motivación laboral medio, el 37,5\% presenta un novel de motivación laboral alto y el 7,5\% presenta un nivel bajo. Este resultado es corroborado por la investigación de Bernable (2018) quien halló que los participantes del estudio presentaban un nivel medio de motivación laboral.

Con relación al compromiso organizacional, el $73,8 \%$ de los trabajadores presentan un nivel de compromiso organizacional alto, lo cual se caracteriza porque se identifican con su organización y las metas que se plantearon y desean mantener su relación con ella, el 22,5\% presenta un nivel de compromiso organizacional medio y sólo el 3,8\% presenta un nivel bajo. Estos resultados coinciden con la investigación de Gonzáles (2015) quien halló que los trabajadores se caracterizan por tener un buen nivel de compromiso organizacional.

En la tabla 3 se halló que existe relación alta, directa y significativa entre la motivación laboral y el compromiso organizacional de los trabajadores de la Corte Superior de Justicia de Madre de Dios, 2017. El coeficiente de correlación rho de Spearman fue de 0,759 con un p-valor de $0,000<0,05$. Esto quiere decir que mientras los trabajadores presenten altos niveles de motivación laboral, tendrán un mayor compromiso hacia su institución y viceversa. Estos resultados coinciden con la investigación de Laura (2015) quien menciona que cuanto más motivado para el trabajo está un trabajador, registrará niveles más altos de engagement. Del mismo modo, coincide con los resultados de la investigación de Rojas y Alegre (2016) quienes hallaron que existe una relación directa y estadísticamente significativa entre la motivación y el compromiso organizacional así como sus dimensiones. Por otra parte, coincide con el trabajo de Córdova (2018) quien estableció que la motivación laboral promueve en los trabajadores al deseo de ser parte de la institución, permanecer como integrante, sentir altos niveles de identificación con la misma y mediante su accionar y ser prescriptor de ésta con su círculo social.

Por otra parte corrobora el hallazgo de Vega (2017) quien halló que existe una relación directa entre motivación laboral y compromiso organizacional en el personal del área de Seguridad de la Información y prevención del Fraude de la empresa Telefónica Ingeniería de seguridad, 2015 debido a que el valor del coeficiente de correlación de Spearman fue 0,296, con un p-valor de 0,009 que resulta menor al valor 0,05 de la significancia. Finalmente guarda relación con el trabajo de García y Gonzáles (2018) quienes demostraron que existe una relación positiva considerable, directa y significativa en la motivación laboral y el compromiso organizacional, ello significa que si la motivación laboral mejoraría también lo haría el compromiso organizacional; en situación contraria, ambas empeorarían, eso es lo que demuestra una relación lineal.

Teniendo en cuenta lo planteado por Marín (2003) el uso de planes de participación en los beneficios, permite incrementar el compromiso con la organización de los trabajadores por los incentivos que esta les otorgue. Es decir, que mientras un trabajador sienta que la empresa está consciente de los beneficios que le produce a la misma, él se sentirá más comprometido con la organización. 
Con relación a la correlación entre la variable motivación y las dimensiones de la variable compromiso organizacional, todas resultaron ser significativas: compromiso afectivo ( $r s=0,652$; $\mathrm{p}=0,000<0,05)$, compromiso de continuidad ( $r s=0,579 ; \mathrm{p}=0,000<0,05)$ y compromiso normativo ( $r s=0,751 ; p=0,000<0,05)$. Estos resultados son corroborados por Chambi (2018) quien encontró que las dimensiones compromiso afectivo, de continuidad y normativo se correlacionan directa y significativamente con la motivación laboral.

Como se puede ver, la motivación es una variable trascendental que influye directamente en el compromiso organizacional, permitiendo que los trabajadores se identifiquen con su institución, asuman los fines y objetivos institucionales como suyos, tengan la plena voluntad de permanecer en ella y se pongan la camiseta institucional al desarrollar sus actividades, logrando de esta manera que se involucren y desempeñen de forma adecuada.

Por tal motivo, es responsabilidad del personal directivo y jerárquico generar o gestionar políticas que incentiven a los trabajadores tales como reconocimientos públicos mediante resoluciones de felicitación, cursos de especialización, bonos e incentivos para que se sientan satisfechos con la labor que realizan y principalmente comprometidos con la institución.

\section{CONCLUSIONES}

El objetivo general de investigación fue alcanzado puesto que se logró determinar que existe una relación alta, directa y significativa entre la motivación laboral y el compromiso organizacional en los trabajadores de la Corte Superior de Justicia de Madre de Dios, 2017. El coeficiente de correlación fue de rho de Spearman fue de 0,759 con un $\mathrm{p}$-valor inferior al nivel de significancia $(\mathrm{p}=0,000<0,05)$.

Del mismo modo, se pudo corroborar la existencia de relación entre la motivación laboral y las dimensiones compromiso afectivo ( $\mathrm{rs}=0,652)$, compromiso de continuidad ( $\mathrm{rs}=0,579)$ y compromiso normativo ( $\mathrm{rs}=0,751)$. En todos los casos las correlaciones fueron significativas $(\mathrm{p}=0,000<0,05)$.

Finalmente, un resultado emergente a la presente investigación fue que se pudo identificar que los trabajadores de la Corte Superior de Justicia de Madre de Dios se caracterizan porque su nivel de motivación laboral se encuentra en un nivel medio y su nivel de compromiso organizacional es alto.

\section{REFERENCIAS BIBLIOGRÁFICAS}

Bernable, B. (2018). Motivación laboral en el compromiso organizacional docente de las instituciones educativas del nivel inicial Red $N^{\circ} 12$-UGEL Nº 06 Vitarte; 2017. (Tesis de Maestría). Universidad César Vallejo. http://repositorio. ucv.edu.pe/bitstream/handle/UCV/21663/Bernable_NBY. pdf? sequence $=1$ \&isAllowed $=y$

Chambi, J. (2018). Relación de la motivación laboral con el compromiso organizacional de los trabajadores de la $\mathrm{Mu}$ nicipalidad Distrital de Paucarpata en el año 2018. (Tesis de Licenciatura), Universidad Nacional de San Agustín de Arequipa. http://bibliotecas.unsa.edu.pe/bitstream/handle/UNSA/7413/RIchtajm.pdf?sequence $=1$ \&isAllowed $=y$ Chiang, M., Gómez, N. y Wackerling, L. (2016). Compromiso Organizacional del Funcionario Municipal Rural de la Provincia de Nuble, Chile. Ciencia \& trabajo, 18(56), 134-138. https://scielo.conicyt.cl/pdf/cyt/v18n56/art10. pdf

Córdova, K. (2018). Motivación laboral y compromiso organizacional en los trabajadores del CETICOS Paita. (Tesis de Licenciatura). Universidad Nacional de Piura. http://repositorio.unp.edu.pe/bitstream/handle/UNP/1426/ADMCOR-COR-2018.pdf? sequence $=1$ \&isAllowed $=y$

Estrada, E., y Mamani, H. (2020). Compromiso organizacional y desempeño docente en las Instituciones de Educación Básica. Revista Innova Educación, 2(1), 132-146. https://doi.org/10.35622/j.rie.2020.01.008

Foncubierta, M. y Sánchez, J. (2019). Hacia la felicidad laboral: atender motivaciones y eliminar «temores digitales». Revista de Ciencias de la Administración y Economía, 8(18), 239-257. https://doi.org/10.17163/ret.n18.2019.04

García, L. y Gonzáles, G. (2018). Motivación laboral y el compromiso organizacional, en los trabajadores de la $\mathrm{Mu}$ nicipalidad Provincial Sánchez Carrión, 2018. (Tesis de Licenciatura). Universidad Peruana Unión. https://repo- 
sitorio.upeu.edu.pe/bitstream/handle/UPEU/1276/Leidy_ Tesis_Titulo_2018.pdf?sequence=5\&isAllowed $=\mathrm{y}$

Gonzáles, W. (2015). Clima, reconocimiento y compromiso laboral de los empleados de Vizcarra y Asociados. (Tesis de Maestría). Universidad de Montemorelos.

Hellriegel, D. y Slocum, J. (2004). Comportamiento Organizacional. Internacional Thomson Editores.

Hernández, R. Fernández, C. y Baptista, P. (2010) Metodología de la Investigación. (6 ${ }^{\mathrm{a} e d .}$.). Mc Graw-Hill.

Herrera, J. y Zamora, N. (2014). ¿Sabemos realmente que es la motivación? Correo Científico Médico de Holguín, 18(1), 126-128. http://www.revcocmed.sld.cu/index.php/ cocmed/article/view/1168/473

Herzberg, F., Mausner, B. \& Snyderman, B. (1959). The motivation to work (2a ed.). Wiley.

Laura, A. (2015). Motivación laboral y engagement. (Tesis de Licenciatura en Contabilidad). Universidad FASTA.

Marín, M. (2003). Relación entre el clima y el compromiso organizacional en una empresa del sector petroquímico. (Tesis de Licenciatura), Universidad Católica Andrés Bello.

Marín, H. y Placencia, M. (2017). Motivación y satisfacción laboral del personal de una organización de salud del sector privado. Horizonte Médico, 17(4), 42-52. https:// doi.org/10.24265/horizmed.2017.v17n4.08

Meyer, J. y Allen, N. (1991). A three component conceptualization of organizational commitment. Human Resource Management Review, 1, 61 - 98.

Neves, T., Graveto, J., Rodrigues, V., Marôco, J. y Parreira, P. (2018). Compromiso organizacional, cualidades psicométricas e invarianza del cuestionario de Meyer y Allen para enfermeros portugueses. Revista Latino-Americana de Enfermagem, 26, 1-11. https://doi.org/10.1590/15188345.2407.3021

Olivares, S. y Gonzáles, M. (2014). Psicología del trabajo. Grupo Editorial Patria.

Pérez, R. (2014). Motivación y Compromiso Organizacional en Personal Administrativo de Universidades Limeñas. (Tesis de Licenciatura). Pontificia Universidad Católica del Perú. http://tesis.pucp.edu.pe/repositorio/bitstream/handle/20.500.12404/5747/PEREZ_TAPIA_RAQUEL_MOTIVACION_PERSONAL.pdf? sequence $=1$ \&isAllowed $=\mathrm{y}$

Rivera, O. (2010). Compromiso organizacional de los docentes de una institución educativa privada de Lima Metropolitana y su correlación con variables demográficas. (Tesis de Maestría). Pontificia Universidad Católica del Perú. http://tesis.pucp.edu.pe/repositorio/bitstream/hand-
le/20.500.12404/4807/RIVERA_CARRASCAL_OSCAR_ COMPROMISO_DEMOGRAFICAS.pdf? sequence $=1 \& \mathrm{i}-$ sAllowed $=\mathrm{y}$

Robbins, S. (2004). Comportamiento Organizacional. Editorial Prentice Hall Hispanoamérica.

Rojas, E. y Alegre, A. (2016). Motivación laboral y compromiso organizacional en colaboradores del área logística de una empresa metálica de Lima. Nuevos Paradigmas, 1(1), $27-42$.

Salazar, J., Guerrero, J., Machado, Y. y Cañedo, R. (2009). Clima y Cultura Organizacional: dos componentes esenciales de la productividad laboral. ACIMED, 20(4), 6775. https://www.medigraphic.com/pdfs/acimed/aci-2009/ aci094d.pdf

Sulca, G. (2015). Impacto de la motivación en la gestión de una organización policial. (Tesis de Maestría). Universidad Nacional Mayor de San Marcos. http://cybertesis.unmsm. edu.pe/bitstream/handle/cybertesis/4437/Sulca_zg.pd$\mathrm{f}$ ? sequence $=1$ \&is Allowed $=\mathrm{y}$

Vega, S. (2017). Motivación laboral y compromiso organizacional en el personal del área de Seguridad de la Información y prevención del Fraude de la empresa Telefónica Ingeniería de seguridad, 2015. (Tesis de Maestría). Universidad César Vallejo. http://repositorio.ucv.edu.pe/bitstream/handle/UCV/7681/Vega_CS.pdf?sequence=1\&isAllowed $=\mathrm{y}$

Vargas, P. (2012). La motivación de logro en emprendedores de negocios culturales de la ciudad de Huamanga-Ayacucho. (Tesis de Licenciatura). Pontificia Universidad Católica del Perú. 